Materials can be strengthened by nanoscale stacking faults

This content has been downloaded from IOPscience. Please scroll down to see the full text. 2015 EPL 11036002

(http://iopscience.iop.org/0295-5075/110/3/36002)

View the table of contents for this issue, or go to the journal homepage for more

Download details:

IP Address: 159.226.199.8

This content was downloaded on 21/07/2015 at 02:35

Please note that terms and conditions apply. 


\title{
Materials can be strengthened by nanoscale stacking faults
}

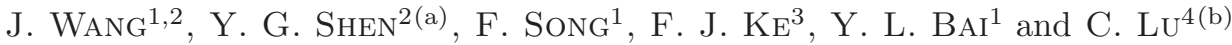 \\ 1 State Key Laboratory of Nonlinear Mechanics (LNM), Institute of Mechanics, Chinese Academy of Sciences \\ Beijing 100190, China \\ 2 Department of Mechanical and Biomedical Engineering, City University of Hong Kong \\ Kowloon, Hong Kong, China \\ 3 School of Physics and Nuclear Energy Engineering, Beihang University - Beijing 100191, China \\ 4 Department of Mechanical Engineering, Curtin University - Perth, WA 6845, Australia
}

received 14 November 2014; accepted in final form 29 April 2015

published online 18 May 2015

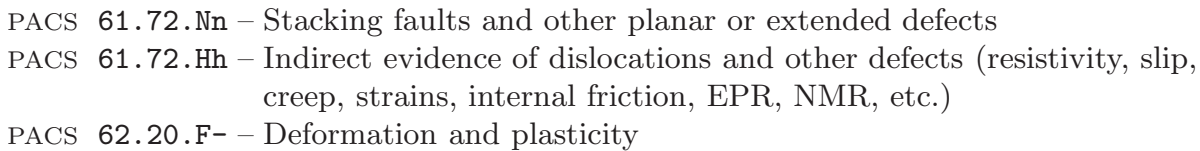

\begin{abstract}
In contrast to the strength of single crystals, stacking faults (SFs) are usually an unfavorable factor that weakens materials. Using molecular-dynamics simulations, we find that parallel-spaced SFs can dramatically enhance the strength of zinc-blende SiC nanorods, which is even beyond that of their single-crystal counterparts. Strengthening is achieved by restricting dislocation activities between nanoscale neighboring SFs and its overall upward trend is dominated by the volume fraction of SFs. The similar strengthening mechanism is also found in face-centeredcubic metals and their alloys. It is more promising than the traditional methods of decreasing nanoscale grains or twins due to the inverse Hall-Petch effect. This study sheds light on the structural design of nanomaterials with high strength.
\end{abstract}

Copyright (C) EPLA, 2015

Stacking faults (SFs) are one or more layer interruptions in the stacking sequence of a crystal lattice. These interruptions carry a certain amount of SF energy and thus weaken the crystal [1]. Moreover, SFs cause the lattice disregistry in neighboring atomic layers, leading to a natural source of dislocations near them. In addition, SFs also participate in dislocation activities when an external load is applied. Attempts to strengthen materials, therefore, seldom resort to SFs. Instead, a variety of closepacked metals engineered with nanoscale grains and twins have been developed to improve their strengths $[2,3]$. The strengthening mechanism by twins in face-centered-cubic (FCC) metals and alloys can be traced back to a pioneer work [4]. It is found out that a cobalt- 8 wt.\% iron alloy was elongated along the orientation of [110], and for the strain propagation associated with a slip band, $\frac{1}{2}[101]$ and $\frac{1}{6}[\overline{1} 12]$ dislocations have to dissociate at the intersections between slip bands and twins. This process creates an extra length of dislocations and consumes more energy. That is, strengthening can be achieved via the rising stress by

\footnotetext{
(a) E-mail: meshen@cityu.edu.hk

(b) E-mail: C.Lu@curtin.edu.au
}

which dislocations cut through twins. In practice, however, it is a big challenge to fabricate ideal twins without introducing disorder that arises from a stacking sequence, suggesting that SFs are more common than twins in metals and alloys. Recently, it has been confirmed that deformation of nanotwinned metals is mixed with activities of SFs [3]. It is obvious that, although the role of twins and grains in strengthening metals has been well investigated, there is still lack of knowledge and understanding on the contribution of SFs.

Similar to metals, SFs that are prevalent in close-packed ceramics and semiconductors can also significantly affect their mechanical and electric properties. For example, both electrostatic and London dispersion interactions in boron nitride were reported to be strongly dependent on the interlay spacing and stacking order, while SFs in $\mathrm{SiC}$ cause a large dispersion of strength and Young's modulus $[5,6]$. Particularly, recent deformation experiments by in situ transmission electron microscopy have confirmed that semiconductor GaAs nanowires and $\mathrm{Mg}$ alloys can be strengthened by nanoscale SFs $[7,8]$. It is elusive, however, whether such a strengthening mechanism is universal in a variety of close-packed materials. To answer this question, 
it is instructive to start with a material with the low SF energy, and then a high density of SFs can be introduced. Thus the relevant strength of such a material can be systematically studied. In contrast to metals and alloys, the $\mathrm{SF}$ energy in zinc-blende $\mathrm{SiC}$ is low and even negative, indicating that $\mathrm{SiC}$ with $\mathrm{SF}$ s is much more stable than a single crystal [9]. To the best of our knowledge, there are still no studies on strengthening $\mathrm{SiC}$ by varying volume fraction $v$ of SFs. In this letter, the underlying strengthening mechanism by parallel-spaced SFs in $\mathrm{SiC}$ has been systematically investigated by molecular-dynamics simulations. To elucidate whether such a strengthening mechanism is suitable for various close-packed materials mediated by nanoscale SFs, FCC metals such as gold $(\mathrm{Au})$, silver $(\mathrm{Ag})$ and copper $(\mathrm{Cu})$ and a typical alloy of $\mathrm{Au}_{0.25} \mathrm{Cu}_{0.75}$ are also examined and compared.

The yield strength $\sigma_{y}$ (hereafter referred to as the strength), a stress at the elastic limit that can be enhanced by nanoscale grains and twins, is normally described by the empirical Hall-Petch relationship, $\sigma_{y}=\sigma_{0}+k / d^{n}$, where $\sigma_{0}$ and $k$ are material-dependent constants; $d$ is the grain size or the distance between twin boundaries and $n$ is an exponent [10]. Based on this relationship, it seems that $\sigma_{y}$ could continuously increase with the decrease of d. By using ångström scale twins to tune dislocation activities, the strength of $\mathrm{Au}$ nanowires could reach almost to their theoretical value [11]. In contrast, both experimental tests and molecular-dynamics simulations confirm that there is a critical grain size or twin space, below which copper becomes softer [3]. Such a phenomenon is ascribed to absorption of dislocations by grain boundaries [11]. It is necessary to clarify, therefore, whether SF-based strengthening in close-packed materials is also limited by a critical size of parallel-spaced SFs.

In a close-packed material, there are three basic sites, denoted as A, B and C, respectively. Zinc-blende structured SiC and FCC metals adopt a periodic ABC stacking sequence. A disordered stacking sequence yields SFs, as shown in fig. 1(a) by dark stripes vertical to the axis of a [111]-oriented SiC nanorod (NR) [12]. Figure 1(b) is a model that contains uniformly distributed SFs, with a parallel spacing of $5 \mathrm{~nm}$ between neighboring SFs (hereafter referred to as the SF parallel spacing $d$ ). In addition, to clarify whether $d$ or $v$ plays a key role in strengthening NRs, non-uniformly distributed SFs with $d$ from 1.3 to $10 \mathrm{~nm}$ are settled into a $\mathrm{SiC} \mathrm{NR}$ (see fig. 1(c), where the average value of $d$ is $4.3 \mathrm{~nm}$ and $v$ is $11.7 \%$ ). Here, $v$ is equal to the ratio of atoms required to build up SFs in a sample. All samples have a round lateral shape with a diameter of $5 \mathrm{~nm}$ and an aspect ratio of $\sim 6: 1$. Periodic and free boundary conditions are applied along axial and lateral directions, respectively.

A potential function consisting of pairwise ( $\mathrm{Si}-\mathrm{C}, \mathrm{Si}-\mathrm{Si}$ and $\mathrm{C}-\mathrm{C}$ ) and three-body covalent $(\mathrm{Si}-\mathrm{C}-\mathrm{Si}$ and $\mathrm{C}-\mathrm{Si}-\mathrm{C})$ interactions is used for the close-packed $\mathrm{SiC}$ ceramics by means of molecular-dynamics simulations [13,14]. This potential performs well in characterizing the energy of (a)

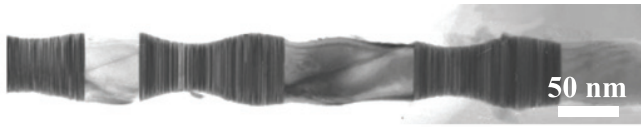

(b)
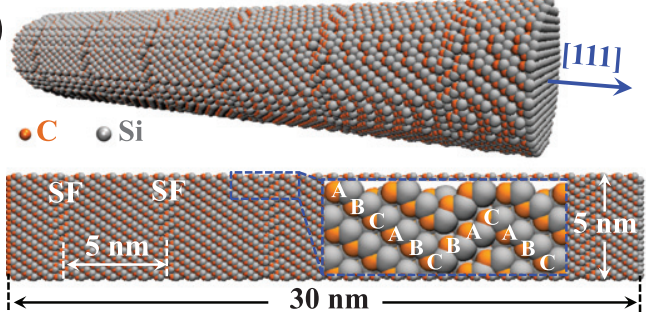

(c)

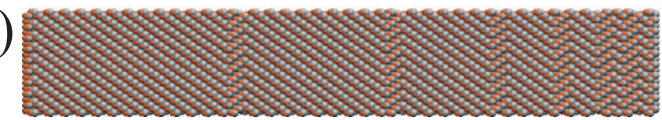

Fig. 1: (Color online) SFs in SiC NRs. (a) A SiC NR adapted from ref. [12], where SFs are stripes perpendicular to the axis in dark regions. (b) Model of a $\mathrm{SiC} \mathrm{NR}$ containing uniformly distributed SFs with $d=5 \mathrm{~nm}$. The inset shows a detailed view of a local SF structure. (c) Sketch of non-uniformly distributed SFs with $d$ ranging from 10 to $1.3 \mathrm{~nm}$.

SFs and SF-dependent mechanical behaviors of SiC polymorphs [14]. Before compression, NRs with various $v$ values of SFs are first relaxed for $20 \mathrm{ps}$ via a modified isothermal-anisobaric ensemble at $300 \mathrm{~K}$ [15]. Then, NRs with a certain $v$ are further relaxed from 20 to 100 ps to get dissimilar initial configurations and velocity distributions. Such a scheme ensures the statistical reliability of calculated mechanical properties. Here, 10 samples for each $v$ are tested and the average value of Young's modulus $E$ (fitted by the elastic regime of a stress-strain curve), strain $\varepsilon_{e}$ at the elastic limit and $\sigma_{y}$ are obtained. To simulate compressive deformation, a modified isothermal-isobaric ensemble is applied to reduce NRs with a strain rate of $-1 \times 10^{-3} \mathrm{ps}^{-1}$ for $1 \mathrm{ps}$ [16]. This is equivalent to a nominal strain of $0.1 \%$. Then, the axial strain is hold for $6 \mathrm{ps}$ via a canonical ensemble to allow stress relaxation. Motions of atoms are integrated by the leapfrog algorithm with a time step of $2 \mathrm{fs}$. The stress tensor is calculated by the classical viral formula and the system temperature is controlled by the Nosé Hoover thermostat [16].

Figure 2(a) shows typical stress-strain curves of $\mathrm{SiC}$ NRs with diverse values of $d$. In a spacing of $30 \mathrm{~nm}$, $\varepsilon_{e}$ is $6.1 \%$. Then, buckling results in an initiation of dislocations in the region between neighboring SFs. These dislocations generated on the compressive side of a NR propagate on the (11̄i) plane along either [011] or [112] orientation, which leads to a stress drop from 19.2 to $11.0 \mathrm{GPa}$. The detected dislocation activities are consistent with experimental observations during bending tests on SiC nanowires [17]. The stress drop in the stressstrain curve implies an event of dislocation nucleation to propagation. The NR is compressed to a strain of $12 \%$ without fracture. Dislocation activities are also detected 
(a)

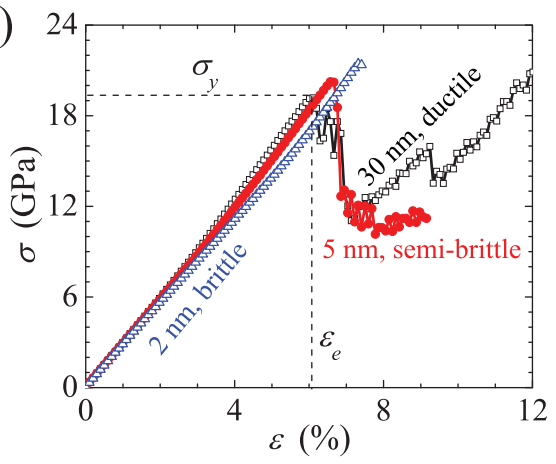

(b)

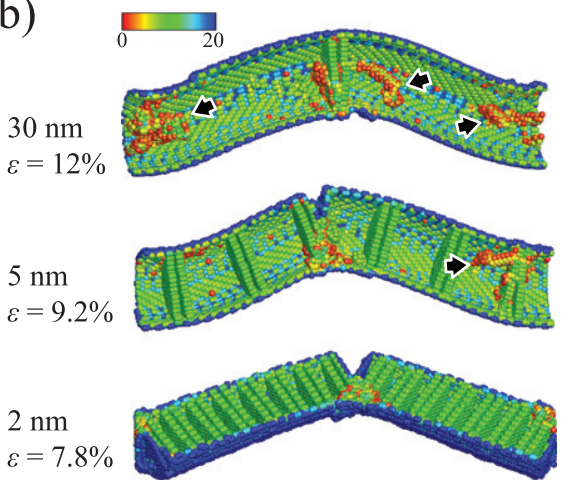

Fig. 2: (Color online) Deformation of $\mathrm{SiC}$ NRs with different SF fractions. (a) Stress-strain curves of SiC NRs with $d$ of 30, 5 and $2 \mathrm{~nm}$, respectively. (b) Beyond the elastic limit, plastic deformation dominates the NR with $d=30 \mathrm{~nm}$ through dislocation activities, where arrowheads indicate dislocation lines. A NR with $d=5 \mathrm{~nm}$ shows a semi-brittle behavior. As $d$ is further reduced to $2 \mathrm{~nm}$, a NR subjects to brittle fracture since dislocation activities are forbidden.

as $d$ decreases from 30 to $5 \mathrm{~nm}$. $\sigma_{y}$ increases from 19.2 to $20.2 \mathrm{GPa}$ and the corresponding $\varepsilon_{e}$ is from 6.1 to $6.6 \%$. Eventually, brittle fracture occurs in the NR through bond breaking between neighboring (111) planes on the tensile side and the strain is up to $9.2 \%$. With $d$ further reducing to $2 \mathrm{~nm}, \sigma_{y}$ and $\varepsilon_{e}$ rebound to $21.4 \mathrm{GPa}$ and $7.3 \%$, respectively. Surprisingly, there are no initiated dislocations and the NR exhibits a pure elastic response with brittle fracture (see fig. 2(b)), where dislocation activities can be evidenced by means of a central symmetry parameter [18].

As illustrated in fig. $3, \sigma_{y}$ of $\mathrm{SiC}$ NRs closely depends on the spacing $d$. It is of interest to note that $\sigma_{y}$ increases by $13 \%$ from $19.1 \pm 0.1$ to $21.5 \pm 0.2 \mathrm{GPa}$ when $d$ decreases from 30 to $2 \mathrm{~nm}$. Obviously, the strength data follow the Hall-Petch relationship and a critical spacing $d$ does not exist, causing the reduction of $\sigma_{y}$. In the SiC NR with nonuniformly distributed SFs, dislocations are active in the region of $d=10 \mathrm{~nm}$. Here it is worth noting that $\sigma_{y}$ of this NR coincides with that of a NR with uniformly distributed SFs with $v=12 \%$. Moreover, $\varepsilon_{e}$ is proportional to $v$, whilst the reverse trend is seen for $E$, as shown in figs. 3(b) and (c), respectively.

Under comparison, the tensile deformation of $\mathrm{SiC}$ NRs with SFs is investigated. Results show that all NRs are (a)

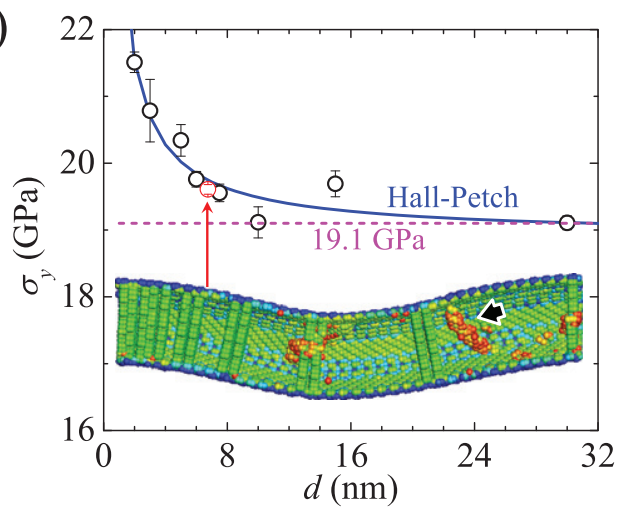

(b)

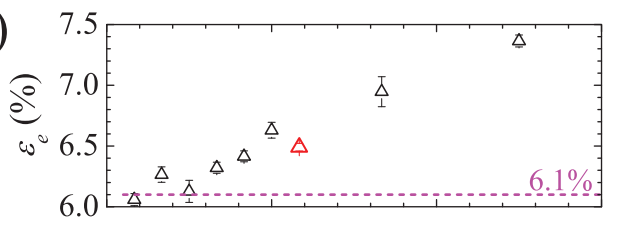

(c)

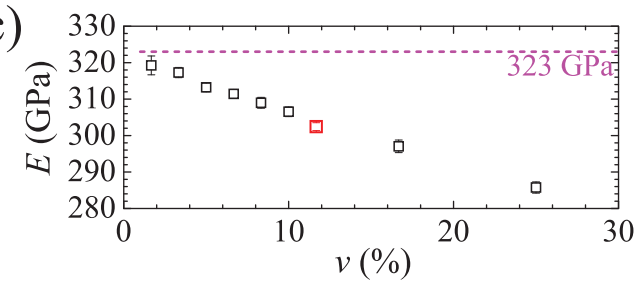

Fig. 3: (Color online) SFs strengthen SiC NRs. (a) $\sigma_{y}$ increases with the decrease of $d$. Inset shows that, in a NR with nonuniformly distributed SFs, dislocation activities are captured from regions with a bigger value of $d$, where an arrowhead denotes a dislocation line. The Hall-Petch curve is fitted by using $n=1$. (b) $\varepsilon_{e}$ is proportional to $v$, and (c) $E$ reduces as $v$ increases. Red circles, triangles and boxes correspond to $\sigma_{y}, \varepsilon_{e}$ and $E$ of the NR with non-uniformly distributed SFs, respectively. Error bars indicate the standard deviation. The dashed lines represent the properties of a single-crystal NR.

subject to brittle fracture at the elastic limit. Fracture origins from the breaking of $\mathrm{Si}-\mathrm{C}$ bonds between neighboring (111) planes. In contrast to compression, however, $\sigma_{y}$ is slightly below that of a single-crystal NR [14]. The influence of the aspect ratio on the compressive deformation of NRs is also analyzed. It is found that both $\sigma_{y}$ and $\varepsilon_{e}$ increase as the aspect ratio reduces, which agrees with the conventional Euler buckling theory. It is worth noting that, however, as the aspect ratio decreases to $4: 1$, buckling can be negligible and a NR follows the dislocation dominated ductility [14].

To ascertain whether a size-dependent mechanical response exists, single-crystal NRs with the diameter $D$ ranging from 2.5 to $10 \mathrm{~nm}$ and the same aspect ratio of $6: 1$ are compressed. It is shown that, due to the surface stress-induced nonlinear elasticity, both $\sigma_{y}$ and $E$ increase with the increase of the diameter $D$ [14]. A similar trend is found in $\mathrm{Ni}_{3} \mathrm{Al}$ nanowires [19]. Further, to study the interplay between the two characteristic dimensions $(d$ and $D)$, 
NRs containing the SFs $(d=5 \mathrm{~nm})$ with $D=5,7.5$ and $10 \mathrm{~nm}$ are simulated. It is shown that, taking single crystals as reference, NRs with SFs yield a stronger $\sigma_{y}$ and they can be continuously reinforced as $d / D$ increases [14].

The simulated results on SiC NRs show that, by localizing dislocation activities in spaces between SFs and preventing their propagation, strengthening is realized. Such a strengthening mechanism is similar to that observed in close-packed metals engineered by nanoscale twins and grains [2]. As shown in fig. 2, the controlled extent of dislocation activities is embodied in ductile, semi-brittle and brittle behaviors of SiC NRs. Hindering the dislocation activity in a material leads to the increase of its strength and the transition from ductile to brittle behavior [20]. A similar phenomenon was also observed in nanotwinned $\mathrm{Au}$ nanowires [5].

The overall strengthening trend for $\mathrm{SiC}$ demonstrates that $\sigma_{y}$ is proportional to $d^{-1}$, which echoes the recent experimental observation on $\mathrm{Mg}$ alloy with SFs [8]. Moreover, an SF can be regarded as a one-layer twin and thus a stress more than the yield strength can be obtained due to the dissociation of dislocations at intersections between slip bands and twins during the plastic deformation [4]. However, to improve the yield strength of a NR, SFs play a key role in postponing the initiation of dislocations (see fig. 3(b), where $\varepsilon_{e}$ is proportional to $v$ ) because they usually occur after the elastic response.

Figure 3 indicates that, in the case of non-uniformly distributed SFs, the value of $\sigma_{y}$ cannot be predicted by either the average $d$ or the maximum $d$. However, $E$ and $\varepsilon_{e}$ always exhibit clear tendencies as $v$ varies. Therefore, it is suitable to describe the overall trend of strengthening by using $v$ rather than $d$ as a variable parameter because non-uniformly distributed SFs are more common.

To understand how $E$ and $\varepsilon_{e}$ vary with $v$, let us assume a NR subjected to an external load along its axis. Here, the NR can be simplified as a serial mechanical system, consisting of two parts with dissimilar constitutive relationships [14]. One part includes a region with SFs and the other without SFs. Thus, $\varepsilon_{e}$ of the NR can be written as

$$
\varepsilon_{e}=\varepsilon_{c}+\left(\varepsilon_{s}-\varepsilon_{c}\right) v
$$

where $\varepsilon_{s}$ is the strain at the elastic limit in the region with SFs and $\varepsilon_{c}$ is the strain in the region without SFs. $E$ of the NR is

$$
E=\frac{E_{c} E_{s}}{E_{s}+\left(E_{c}-E_{s}\right) v},
$$

where subscripts $s$ and $c$ indicate the mechanical values in regions with and without SFs, respectively (see the Supplemental Material as noted in ref. [14] for a detailed derivation). Then, $\sigma_{y}$ can be expressed as a function of $v$, that is

$$
\sigma_{y}=\frac{E_{c} E_{s}\left[\varepsilon_{c}+\left(\varepsilon_{s}-\varepsilon_{c}\right) v\right]}{E_{s}+\left(E_{c}-E_{s}\right) v}
$$

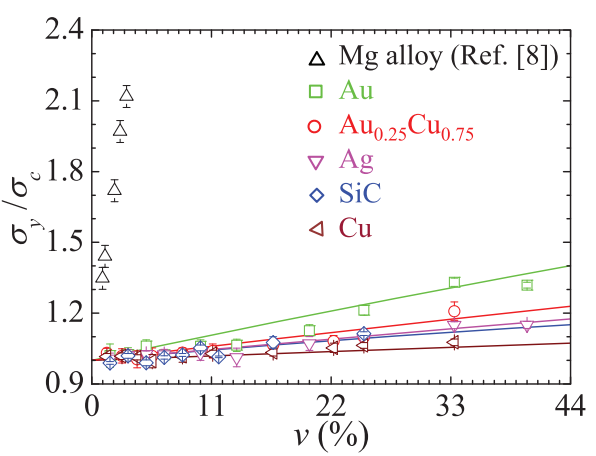

Fig. 4: (Color online) Strength of materials varies with $v$. For comparison, strength is also rescaled by $\sigma_{c}=\varepsilon_{c} E_{c}$. Triangular $(\triangle)$ points are experimental results of $\mathrm{Mg}$ alloy [8], while other scattered points are simulated results. Solid lines are the curves fitted by eq. (3) with parameters as listed in table 1 .

Table 1: Parameters for SiC, FCC metals and an $\mathrm{Au}_{0.25} \mathrm{Cu}_{0.75}$ alloy, which are obtained by fitting eq. (3).

\begin{tabular}{ccccc}
\hline Material & $E_{c}(\mathrm{GPa})$ & $E_{s}(\mathrm{GPa})$ & $\varepsilon_{c}(\%)$ & $\varepsilon_{s}(\%)$ \\
\hline $\mathrm{SiC}$ & 322.0 & 213.0 & 6.0 & 11.6 \\
$\mathrm{Au}$ & 89.7 & 74.1 & 3.4 & 7.5 \\
$\mathrm{Ag}$ & 92.1 & 81.0 & 5.0 & 7.8 \\
$\mathrm{Cu}$ & 144.9 & 132.5 & 6.4 & 8.1 \\
$\mathrm{Au}_{0.25} \mathrm{Cu}_{0.75}$ & 158.5 & 140.1 & 4.4 & 7.4 \\
\hline
\end{tabular}

As shown in fig. 4, simulated results can be well fitted by eq. (3) with the fitting parameters as listed in table 1. It is obvious that $\sigma_{y}$ monotonously varies with $v$. Strengthening a material by SFs is, therefore, not limited by the inverse Hall-Petch effect [21]. Here it is worth noting that eqs. (1)-(3) are similar to the mixing rule in composites.

In summary, molecular-dynamics simulations have been performed to investigate the influence of SFs on strength of zinc-blende $\mathrm{SiC}$. It is shown that, by localizing dislocation activities in space between SFs, SiC NRs are strengthened. Strength is a monotone function of the SF fraction, which implies that the strength increase of a material is not limited by a critical value of the SF parallel spacing. Such a mechanism is also suitable for strengthening FCC metals and their alloys. This finding is of importance because it provides a recipe for the structural design of materials with superior mechanical properties.

This work has been supported by the National Natural Science Foundation of China (Grant Nos. 11172024, 11232013, 11372022), the National Basic Research Program of China (2012CB937500), the Opening Fund of State Key Laboratory of Nonlinear Mechanics, the Research Grant Council of the Hong Kong Special Administrative Region, China (Project No. 9041679 (CityU 120611)), the Scientific Research Foundation for the Returned Overseas Chinese Scholars, State Education Ministry, and the Australian Research Council 
(Grant No. DP0985450). Computations were supported by resources provided by the Pawsey Supercomputing Centre with funding from the Australian Government and the Government of Western Australia and the ScGrid of Supercomputing Center, Computer Network Information Center of the Chinese Academy of Sciences.

\section{REFERENCES}

[1] Sun F. et al., Adv. Eng. Mater., 15 (2013) 1034.

[2] LU K. et al., Science, 324 (2009) 349; WANG Y. M. et al., Phys. Rev. Lett., 105 (2010) 215502.

[3] Lu L. et al., Science, 323 (2009) 607; Li X. Y. et al., Nature (London), 464 (2010) 877.

[4] Mahajan S. et al., Acta Metall., 21 (1973) 173.

[5] Constantinescu G. et al., Phys. Rev. Lett., 111 (2013) 036104.

[6] Wang J. et al., EPL, 95 (2011) 63003; Nanotechnology, 23 (2012) 025703; Cheng G. et al., Nano Lett., 14 (2014) 754; Zhang T. Y. et al., J. Appl. Phys., 103 (2008) 104308.

[7] Chen B. et al., Nano Lett., 13 (2013) 4369.

[8] Jian W. W. et al., Appl. Phys. Lett., 103 (2013) 133108; JiAn W. W. et al., Mater. Res. Lett., 1 (2013) 61.

[9] Lindefelt U. et al., Phys. Rev. B, 67 (2003) 155204; Menl M. J. et al., Phys. Rev. B, 61 (2000) 4894.
[10] Hall E. O., Proc. Phys. Soc. B, 64 (1951) 747; Petch N. J., J. Iron Steel Inst., 174 (1953) 25.

[11] Wang J. W. et al., Nat. Commun., 4 (2013) 1742.

[12] Zhang Y. F. et al., Adv. Funct. Mater., 17 (2007) 3435.

[13] Vashishta P. et al., J. Appl. Phys., 101 (2007) 103515. Calculations were conducted by using the DL_POLY2.20 package. For details, see Sмiтh W. et al., Mol. Simul., 28 (2002) 385 .

[14] Supplementary Material for this paper is available via the following link: https://drive.google.com/file/ d/OBymYvQ86SmIxaDR1ZUhrQ1VMOG8/view?usp=sharing.

[15] Melchionna S. et al., Mol. Phys., 78 (1993) 533; Spearot D. et al., Acta Mater., 53 (2005) 3579. A similar technique was established to analyze ferroelectric behaviors of barium titanate. For details, see WANG J. et al., EPL, 92 (2010) 17006.

[16] Nosé S., Mol. Phys., 52 (1984) 255; Hoover W. G., Phys. Rev. A, 31 (1985) 1695; Toxvaerd S., Mol. Phys., 72 (1991) 159.

[17] Han X. D. et al., J. Appl. Phys., 98 (2005) 124307; Han X. D. et al., Nano Lett., 7 (2007) 452.

[18] Kelchner C. L. et al., Phys. Rev. B, 58 (1998) 11085.

[19] Wang Y. J. et al., Appl. Phys. Lett., 102 (2013) 041902.

[20] VAliev R., Nature (London), 419 (2002) 887 and references therein.

[21] Carlton C. E. et al., Acta Mater., 55 (2007) 3749 and references therein. 